\title{
Modular titanium alloy neck failure in total hip replacement: analysis of a relapse case
}

\author{
Marco Ceretti ${ }^{1, *}$ and Francesco Falez ${ }^{2}$ \\ 1 Saint Filippo and Nicola Hospital, Via Adolfo Omodeo 31 d, CAP 00179 Rome, Avezzano, Italy \\ 2 Saint Spirit Hospital, CAP 00193 Rome, Italy
}

Received 15 January 2016, Accepted 23 January 2016, Published online 29 April 2016

\begin{abstract}
Modular neck hip prosthesis born in the 1990 with the aim of allowing the surgeon to modify CCD angle, offset and femoral anteversion intra-operatively restoring patient's original biomechanics. In order to achieve the best biomechanics of the reconstructed hip, preoperative planning is essential. In the last few years modularity has been questioned and an argument made for the return to mono block stems due to events of breakage or disconnection of modular components. Fretting or crevice corrosion may lead to failure of such modular device due to the contamination inside the modular coupling or to high loads. We present a case of repetitive modular femoral neck prosthesis fracture.
\end{abstract}

Key words: Modular neck, Prosthesis, Fatigue fracture, Total hip arthroplasty.

\section{Introduction}

Modularity introduction in the last 1990 has changed the approach strategies in primary and revision total hip replacement. Neck modularity guarantees in primary total hip replacement more options to reproduce the physiological longitudinal and lateral offset and anteversion or retroversion. In total hip revision surgery, modularity has the most important role to adapt the old femoral implant to the new acetabular implant varying offsets with also the stability benefit of the overall system. Fracture of the neck is a risk in neck modularity, which many authors and those in industry are trying to resolve.

\section{Case report}

A 43 -year-old woman (BMI $38.6 \mathrm{~kg} / \mathrm{m}^{2}$, weight $110 \mathrm{~kg}$, height $170 \mathrm{~cm}$ ) came to our observation in October 2006 with right hip pain. Clinical findings and radiograph imaging revealed right hip arthrosis and a varus short neck

In November 2006, the patient underwent right total hip arthroplasty (THA), which was performed with the Hardinge lateral approach. The implant chosen was a modular uncemented Metha titanium stem (Aesculap Orthopaedics) with a $135^{\circ}$ neck long, a $36 \mathrm{~mm}$ medium ceramic head (Biolox delta), a $50 \mathrm{~mm}$ Delta PF cup (Lima Corporate), and Liner in ceramic

\footnotetext{
*Corresponding author: dr.ceretti@gmail.com
}

(Biolox delta). We also put a cerclage for a little crack at the lesser trochanter level.

The patient's initial postoperative course was uncomplicated. After six months, HHS was 98 points and ROM was: flexion $110^{\circ}$, abduction $45^{\circ}$, extension $10^{\circ}$, internal rotation $15^{\circ}$, external rotation $15^{\circ}$.

After two years and five months, the patient came back with atraumatic right hip pain described as unremitting and was associated with grinding and clicking sensation during ambulation with an instability feeling.

X-rays demonstrated deformity at the neck-stem junction with increased neck varus angulation suggestive of a femoral neck fatigue fracture (Figure 1).

The patient was taken to the operating room for revision surgery. During the procedure it was possible to remove a part of the fractured femoral neck from the hip stem. We removed all the femoral implant via the Hardinge approach and it was decided to implant a modular uncemented Modulus titanium hip stem (Lima Corporate, diameter $21 \mathrm{~mm}$ taper B) with titanium long $135^{\circ}$ neck and a $36 \mathrm{~mm}$ medium ceramic femoral head (Figure 2).

The patient's postoperative course was uncomplicated.

After six months, HHS was 88 points and ROM was: flexion $110^{\circ}$, abduction $40^{\circ}$, extension $10^{\circ}$, internal rotation $15^{\circ}$, external rotation $15^{\circ}$. After two years and six months, the patient felt the same non-traumatic right hip pain. X-rays demonstrated the stem rupture at the neck-stem junction (Figure 3). 


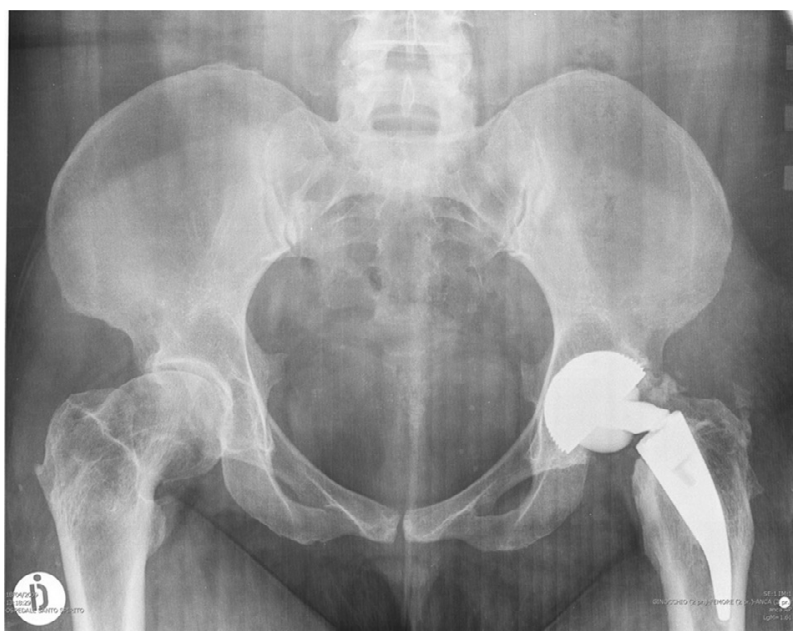

Figure 1. Neck-stem junction breaking in the absence of traumatic injury after two years from the 1st THA (Total Hip Arthroplasty). The prosthesis is a Metha stem implant with modular neck. It was implanted in 2006 when the corrosion theory was not known and the modular neck was in titanium without $\mathrm{CrCo}$.

Our strategy was to remove the modulus hip stem using a Wagner osteotomy and our choice was to implant a Wagner monoblock uncemented titanium stem (Wagner SL revision produced by Zimmer) with a $36 \mathrm{~mm}+3.5$ Biolox delta ceramic head (Figure 4).

\section{Discussion}

Modular hip systems offer the surgeon the potential to restore normal hip biomechanics with the ability to independently adjust offset, version, and limb length.

Fracture of the femoral component is a rare complication in total hip arthroplasty. Before the introduction of cobalt chromium molybdenum and titanium alloys, Charnley estimated the stem fracture prevalence to be $0.23 \%$ with other designs.

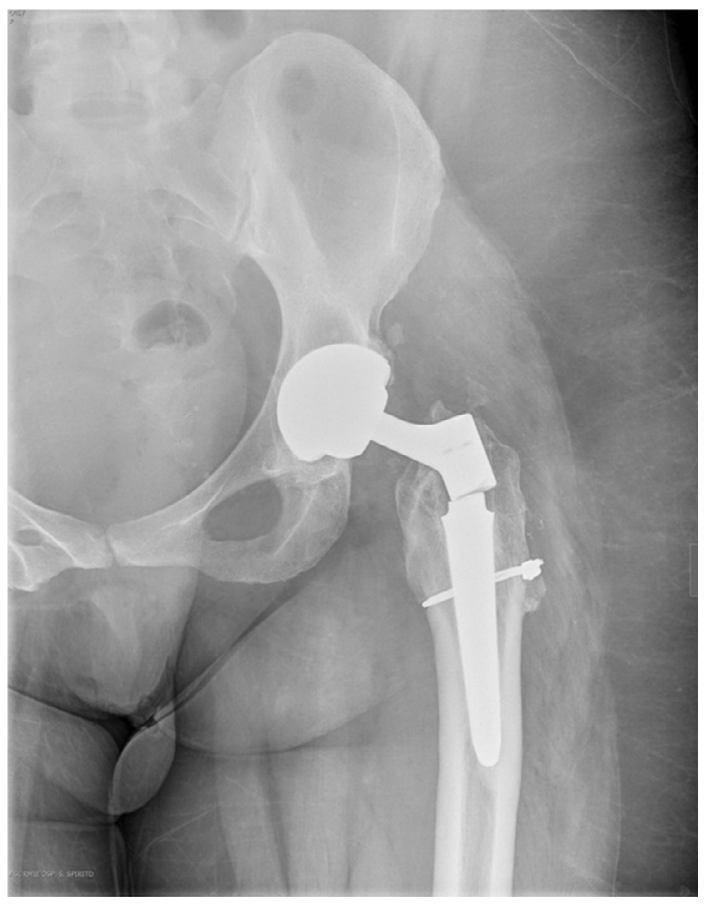

Figure 3. Neck-stem junction breaking in 1st revision implant after atraumatic pain two years after the revision. The rupture is localized on the thread at the neck-stem junction. The neck was virus $\left(135^{\circ}\right)$ in Ti6Al4V.

Modular titanium alloy neck fracture in Metha prosthesis is $1.4 \%$ and the higher incidence of failure is between nine and 42 months postoperatively. There is also a direct proportionality between patient weight, caput-collum-diaphysis (CCD) angle, and failure incidence [1].

In vitro studies of femoral components with neck-stem modularity have shown that corrosion and fretting can occur at the neck-stem junction [2, 3]. Corrosion and fretting occur at both the head-neck and the neck-stem modular junctions [4] and neck-stem junction degradation is more significant than
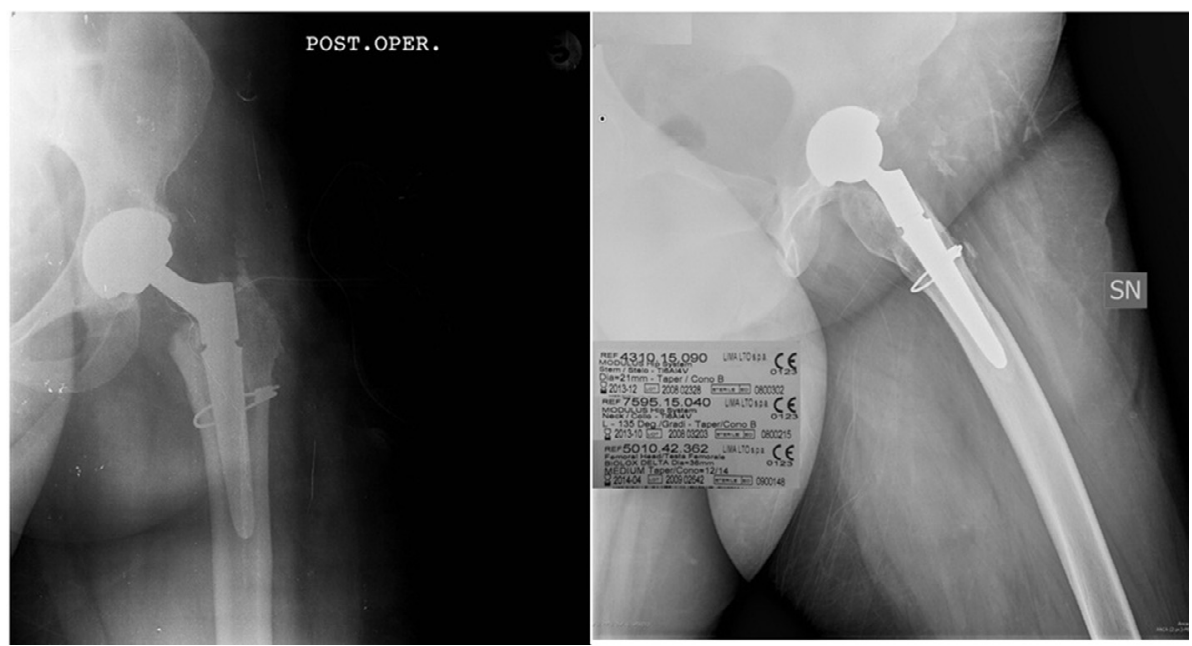

Figure 2. Postoperative X-ray after the Metha stem revision. The 1st implant revision was performed with Lima Modulus implant. Implant details: modulus stem $21 \mathrm{~mm}$ diameter, neck Ti6A14V $135^{\circ}$ taper B, Femoral Head $36 \mathrm{~mm}$ Biolox delta. 


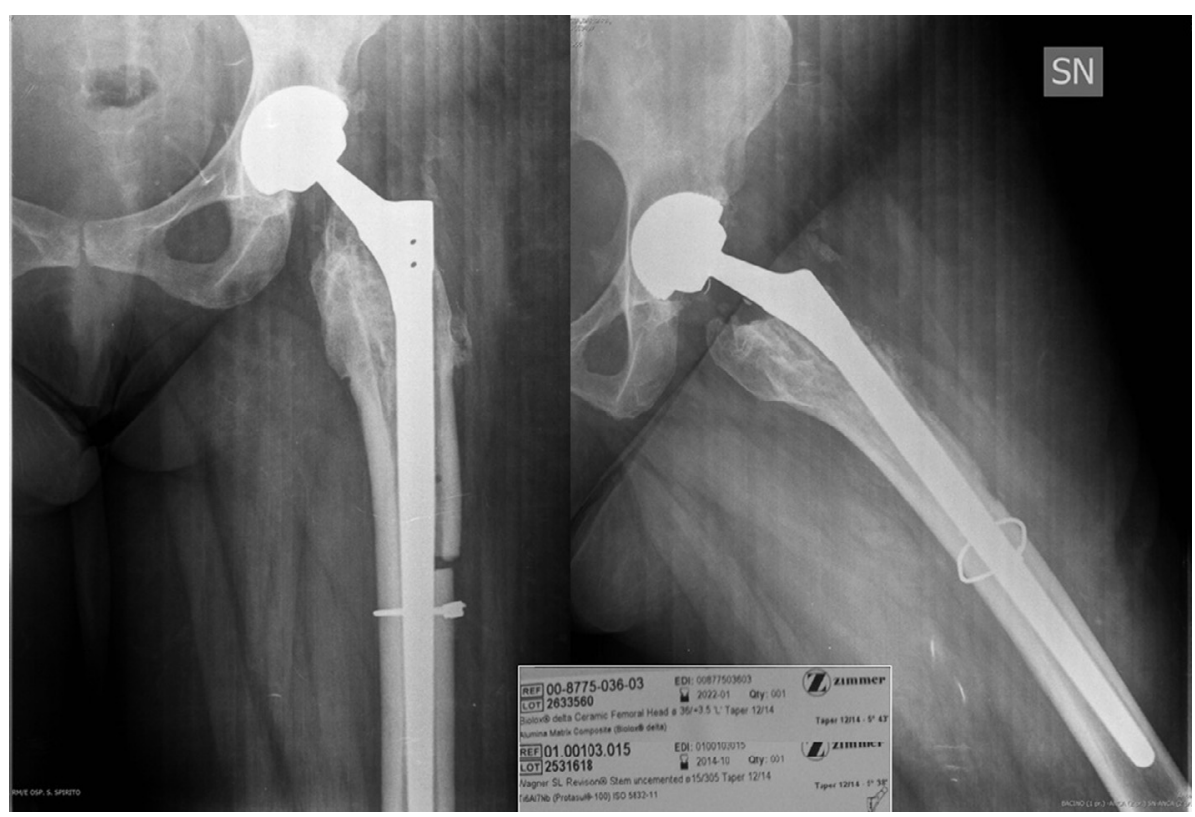

Figure 4. Postoperative X-ray after 2 nd revision. The stem choice was a monolithic Wagner revision implant with the following details: femoral head $36+3.5$, Lima Wagner S1 revision stem. The Wagner stem is already hole.

at the head-neck junction and is believed to be secondary to the increased lever arm and high mechanical stress [2, 3]. Varus neck with increased offset and reduced length is the modular neck style, which creates the highest strain at the modular neck-stem junction and is similar to the geometry reported in other case reports of modular neck failure [5].

Specifically the use of a long varus neck increases the bending moment by $32.7 \%$ compared with the standard short varus neck with increasing stress concentration at the modular junction [6]. Crevice and fretting corrosion are higher in titanium alloy adapters compared with cobalt chrome [1].

Most reported fatigue fractures of modular stems have been associated with titanium (Ti6Al4V) alloy neck and stem components [1].

Micro-motions at the junctional interface induce fretting and crevice corrosion, contributing to micro-crack creation within the zone of corrosion and increasing the risk of dynamic fatigue fracture.

With the increased stresses on the modular head-neck and neck-stem junctions, oxide layers are disrupted, leading to a vicious cycle with the recurrent attempts of the metal to repassivate their surface, subsequently depleting available oxygen supply releasing chloride ions within the joint and lowering local $\mathrm{pH}$.

This creates an anaerobic and acidic environment more conducive to further abrasive wear and corrosion, potentiating the risk of component fracture over time $[1,7,8]$. The combined effects of corrosion, large femoral head components with long modular necks, metal-on-metal components, patient obesity, and activity level may create a local microenvironment that can initiate and perpetuate fatigue failure. Intra-operative contamination of the cone connection with bone particles has a considerable impact on the magnitude of fretting in the interface due to the increased micro-motions [9].
In conclusion, we have formed the opinion that in the presence of a high BMI, a CCD angle $<135^{\circ}$, and a high functional demand the risk of failure is significantly increased in modular prostheses. We also believe that the modularity is not to be discouraged in an absolute manner, rather, if one chooses to go this route in such patients the preferred neck should be CoCrMo rather than Ti6Al4V.

\section{Conflict of interest}

Dr. Ceretti does not have relevant financial relationships to disclose.

Dr. Falez is a paid consultant for Smith \& Nephew, Lima, Samo, and DePuy; receives payment for lectures from Smith \& Nephew, Lima, and DePuy; receives royalties from Smith \& Nephew and receives payment for the development of educational presentations from Smith \& Nephew, Lima, and DePuy.

The authors declare that there is no conflict of interest regarding the publication of this manuscript.

\section{References}

1. Grupp TM, Weik T, Bloemer W, Knaebel HP (2010) Modular titanium alloy neck adapter failures in hip replacement - failure mode analysis and influence of implant material. BMC Musculoskelet Disord 11, 3.

2. Viceconti M, Baleanim M, Squarzoni S, Toni A (1997) Fretting wear in a modular neck hip prosthesis. J Biomed Mater Research 35, 207-216.

3. Viceconti M, Ruggeri O, Toni A, Giunti A (1996) Design related fretting wear in modular neck hip prosthesis. J Biomed Mater Research 30, 181-186. 
4. Kop AM, Swarts E (2009) Corrosion of hip stem with a modular neck taper junction: a retrieval study of 16 cases. J Arthroplasty 24, 1019-1023.

5. Sotereanos NG, Sauber TJ, Tupis TT (2013) Modular femoral neck fracture after primary total hip arthroplasty. J Arthroplasty 28, 196.e7-196.e9.

6. Skendzel JG, Blaha JD, Urquhart AG, et al. (2011) Total hip arthroplasty modular neck failure. J Arthroplasty 26, 338.

7. Urban RM, Jacobs JJ, Gilbert JL, et al. (1994) Migration of corrosion products from modular hip prostheses. Particle microanalysis and histopathological findings. J Bone Joint Surg Am 76, 1345.

8. Gilbert JK, Buckley CA, Jacobs JJ, et al. (1994) Intergranular corrosion fatigue failure of cobalt alloy femoral stems. A failure analysis of two implants. J Bone Joint Surg Am 76, 110.

9. Morlock MM, Grupp TM (2013) The influence of contact conditions and micro motions on the fretting behavior of modular titanium alloy taper connections. Med Eng Phys 35(5), 676-683.

Cite this article as: Ceretti M \& Falez F (2016) Modular titanium alloy neck failure in total hip replacement: analysis of a relapse case. SICOT J, 2, 20 